\title{
On Odds Generalized Exponential-Power Lomax Distribution
}

\author{
Salwa Mahmoud Assar \\ Mathematical Statistics, Cairo University, Institute of Statistical Studies and Research, Giza, Egypt
}

Article history

Received: 23-05-2018

Revised: 26-05-2018

Accepted: 9-06-2018

Email: salwaassar@yahoo.com

\begin{abstract}
A new distribution, called odds generalized exponential-power Lomax distribution is suggested for modeling lifetime data. Some structural properties of the new distribution including; quantiles, Rényi entropy, moments and distribution of order statistics are provided. The model parameters of the new distribution are estimated by the maximum likelihood method. Finally, applications to two real data sets are analyzed to illustrate the importance of the new distribution compared with some known distributions.
\end{abstract}

Keywords: Power Lomax Distribution, T-X Family, Odds Function, Moments, Maximum Likelihood Estimators

\section{Introduction}

The Lomax or Pareto II distribution was introduced originally for modeling business failure data by Lomax (1954). Moreover it has been widely applied in a variety of contexts such as; income and wealth inequality, size of cities, actuarial science, medical and biological sciences. Hassan and Al-Ghamdi (2009) mentioned that it can be applied in reliability modeling and life testing. In the literature, some extensions of the Lomax distribution are available such as Marshall-Olkin extended-Lomax (Ghitany et al., 2007; Gupta et al., 2010), the exponentiated Lomax (Abdul-Moniem, 2012), beta-Lomax, KumaraswamyLomax and McDonald-Lomax (Lemonte and Cordeiro, 2013) and gamma-Lomax (Cordeiro et al., 2013), the transmuted exponentiated Lomax (Ashour and Eltehiwy, 2013), the extended Poisson-Lomax (Al-Zahrani, 2015), exponential Lomax (El-Bassiouny et al., 2015), Weibull Lomax (Tahir et al., 2015a) and exponentiated WeibullLomax (Hassan and Abd-Allah, 2018).

Rady et al. (2016) proposed one of these extensions of the Lomax distribution and referred to as a Power Lomax (PL) distribution with three-parameter $\alpha, \beta$ and $\gamma$. The probability density function (pdf) and cumulative distribution function (cdf) of PL distribution are given, respectively by:

$$
\begin{aligned}
& g(x ; \alpha, \beta, \gamma)=\frac{\alpha \beta}{\gamma} x^{\beta-1}\left(1+\frac{x^{\beta}}{\gamma}\right)^{-(\alpha+1)} ; x>0, \alpha, \beta, \gamma>0, \\
& G(x ; \alpha, \beta, \gamma)=1-\left(1+\frac{x^{\beta}}{\gamma}\right)^{-\alpha},
\end{aligned}
$$

where, $\alpha$ and $\beta$ are shape parameters and $\gamma$ is a scale parameter.

In recent years, there has been a great interest among statisticians and applied researchers in constructing flexible distribution to facilitate better modeling of lifetime data in various situations. Several methods have been developed for generating new family of lifetime distributions. The T-X family is one of the generalized family of distributions which was proposed by Alzaatreh et al. (2013) with the following cdf:

$$
F(x)=\int_{0}^{W(G(x))} r(t) d t,
$$

where, the random variable $T$ called the transformer has pdf $r(t)$ defined on $[a, b]-\infty \leq a<b \leq \infty$ and $W(G(x))$ be a function of $G(x)$.

As a result, many new distributions have come up and studied. Tahir et al. (2015b) proposed a new class of distributions called the odd generalized exponential (OGE) family and study each of the OGE- Weibull distribution, the OGE-Fréchet distribution and the OGEnormal distribution. These models are flexible because of the hazard shapes: increasing, decreasing, bathtub and upside subset of down bathtub. Rosaiah et al. (2016) proposed a new lifetime model, called the odd generalized exponential log logistic distribution and Hassan et al. (2018) suggested a new distribution, called odds generalized exponential-inverse Weibull distribution for modeling lifetime data.

Silva et al. (2017) proposed a new generator of continuous distributions with one extra positive parameter called the odd Lindley-G family. They discussed estimation of the model parameters by 
maximum likelihood and provided an application to a real data set. Haq and Elgarhy (2018) proposed a new generator from Frèchet random variable that is known as the odd Frèchet-G (OFr-G) family of distributions and the maximum likelihood equations are derived for OFr-G family parameters. Also, Hosseini et al. (2018) introduced the generalized odd gamma-G distribution. In particular, $G$ has been considered as the uniform distribution and some statistical properties have been calculated.

The main motivation of this study is to introduce a more flexible model with increasing, decreasing, constant and reversed- $\mathrm{J}$ shaped hazard rate and at the same time provide consistently better fits than other well known-distributions. We provide and study a new form for Power Lomax distribution with three parameters. We call the new distribution; the odds generalized exponential-Power Lomax (OGE-PL) distribution, which is a particular case of T-X family of distributions. The rest of the paper contains the following sections. The new distribution is provided in Section 2. Some statistical properties are given in Section 3. Then, in Section 4, maximum likelihood estimators are obtained. An application of the OGE-PL model to real data set is presented in Section 5. At the end, concluding remarks are addressed in Section 6.

\section{The OGE-PL Distribution}

In this section, the pdf, cdf, reliabilty function, hazard rate function (hrf), reversed-hazard rate function and cumulative hazard rate function of OGE-PL distribution are derived. Expansions for its pdf and cdf are also provided.
We obtain the OGE-PL distribution by considering the exponential distribution as transformer in cdf (3); also, taking; $W(G(x))=\frac{G(x)}{\bar{G}(x)}$, the odds ratio of Power Lomax distribution defined in (2) and $\bar{G}(x)=1-G(x)$ as follows:

$$
F(x ; \alpha, \beta, \gamma, \lambda)=\int_{0}^{\frac{G(x)}{1-G(x)}} \lambda e^{-\lambda t} d t=\int_{0}^{\left(1+\frac{x^{\beta}}{\gamma}\right)^{\alpha}-1} \lambda e^{-\lambda t} d t .
$$

Hence, the cdf of OGE-PL distribution is as follows:

$$
F(x ; \alpha, \beta, \gamma, \lambda)=1-e^{-\lambda\left(\left(1+\frac{x^{\beta}}{\gamma}\right)^{\alpha}-1\right)}, x>0 .
$$

The corresponding pdf is obtained as follows:

$$
\begin{gathered}
f(x ; \alpha, \beta, \gamma, \lambda)=\frac{\alpha \beta \lambda}{\gamma} x^{\beta-1}\left(1+\frac{x^{\beta}}{\gamma}\right)^{\alpha-1} e^{-\lambda\left(\left(1+\frac{x^{\beta}}{\gamma}\right)^{\alpha}-1\right)}, \\
x>0, \alpha, \beta, \gamma \text { and } \lambda>0
\end{gathered}
$$

Plots of the pdf of OGE-PL distribution for some selected parameter values are displayed in Fig. 1. As seems from this figure, the pdf of OGE-PL distribution can be symmetric, unimodel and right skewed according to the selected values of parameters.

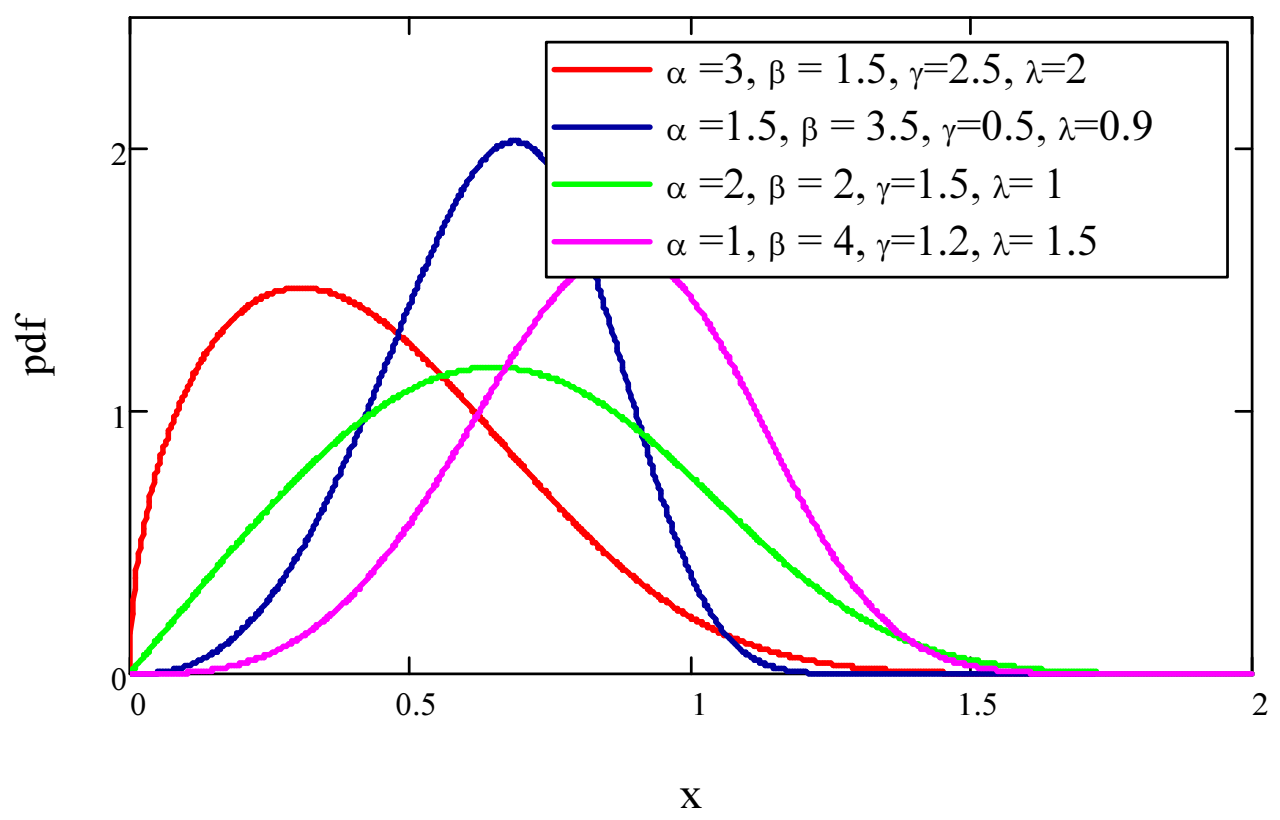

Fig. 1: Plots of the pdf of OGE-PL distribution for selected values of the parameters 


\section{Useful Expansions for OGE-PL Distribution}

Firstly, an expansion for pdf is derived. The power series for the exponential function in pdf (5) can be written as follows:

$e^{-\lambda\left(\frac{G(x ; \xi)}{\bar{G}(x ; \xi)}\right)}=\sum_{i=0}^{\infty} \frac{(-1)^{i} \lambda^{i}}{i !}\left(\frac{G(x ; \xi)}{\bar{G}(x ; \xi)}\right)^{i}$,

where, $\xi=(\alpha, \beta, \gamma)$. Then, by inserting expansion (6) in pdf (5), we have:

$$
\begin{gathered}
f(x ; \alpha, \beta, \gamma, \lambda)=\frac{\alpha \beta \lambda}{\gamma} x^{\beta-1}\left(1+\frac{x^{\beta}}{\gamma}\right)^{\alpha-1} \\
\sum_{i=0}^{\infty} \frac{(-1)^{i} \lambda^{i}}{i !}\left(\frac{G(x ; \xi)}{\bar{G}(x ; \xi)}\right)^{i}
\end{gathered}
$$

By using the generalized binomial expansion, then:

$$
\bar{G}(x ; \xi)^{-i}=\sum_{j=0}^{i} \frac{\Gamma(i+j)}{j ! \Gamma(i)} G(x ; \xi)^{j} .
$$

Inserting (8) in (7), the OGE-PL density function can be expressed as follows:

$$
f(x ; \alpha, \beta, \gamma, \lambda)=\frac{\alpha \beta \lambda}{\gamma} x^{\beta-1}\left(1+\frac{x^{\beta}}{\gamma}\right)^{\alpha-1} \sum_{i=0}^{\infty} \sum_{j=0}^{i} \frac{(-1)^{i} \lambda^{i} \Gamma(i+j)}{i ! j ! \Gamma(i)} G(x ; \xi)^{i+j} .
$$

So, the expansion form of pdf is as follows:

$$
\begin{aligned}
& f(x ; \alpha, \beta, \gamma, \lambda)=\frac{\alpha \beta \lambda}{\gamma} x^{\beta-1}\left(1+\frac{x^{\beta}}{\gamma}\right)^{\alpha-1} \\
& \sum_{i=0}^{\infty} \sum_{j=0}^{i} c_{i, j}\left(1-\left(1+\frac{x^{\beta}}{\gamma}\right)^{-\alpha}\right)^{i+j},
\end{aligned}
$$

where, $c_{i, j}=\frac{(-1)^{i} \lambda^{i} \Gamma(i+j)}{i ! j ! \Gamma(i)}$.

Again, by using the binomial expansion for the last term in (9), we have the final expansion form of pdf as follows:

$$
\begin{aligned}
f(x ; \alpha, \beta, \gamma, \lambda) & =\sum_{i=0}^{\infty} \sum_{j=0}^{i} \sum_{k=0}^{i+j} \eta_{i, j, k} g(x ; \alpha(k-1), \beta, \gamma), \\
x & >0 ; \alpha, \beta, \gamma \text { and } \lambda>0,
\end{aligned}
$$

where,

$$
\eta_{i, j, k}=\frac{(-1)^{k+i} \lambda^{i+1} \Gamma(i+j)}{(k-1) i ! j ! \Gamma(i)}\left(\begin{array}{c}
i+j \\
k
\end{array}\right)
$$

$g(x ; \alpha(k-1), \beta, \gamma)$ is the pdf of PL distribution defined in (1) with parameters $a(k-1), \beta$ and $\gamma$.

\section{Reliability Analysis}

This subsection gives expressions for the reliability function, hazard function, reversed hazard function and cumulative hazard rate function for the proposed model.

The reliability function and hrf of the OGE-PL distribution are respectively given by:

$$
\begin{gathered}
\bar{F}(x ; \alpha, \beta, \gamma, \lambda)=\exp \left(-\lambda\left(\left(1+\frac{x^{\beta}}{\gamma}\right)^{\alpha}-1\right)\right), \\
h(x ; \alpha, \beta, \gamma, \lambda)=\frac{\alpha \beta \lambda}{\gamma} x^{\beta-1}\left(1+\frac{x^{\beta}}{\gamma}\right)^{\alpha-1} .
\end{gathered}
$$

Figure 2 gives the plots of the hrf of OGE-PL distribution for some selected parameter values. Figure 2 indicates that OGE-PL hrfs can be increasing, decreasing and constant. This fact implies that the OGE-PL can be very useful for fitting data sets with various shapes.

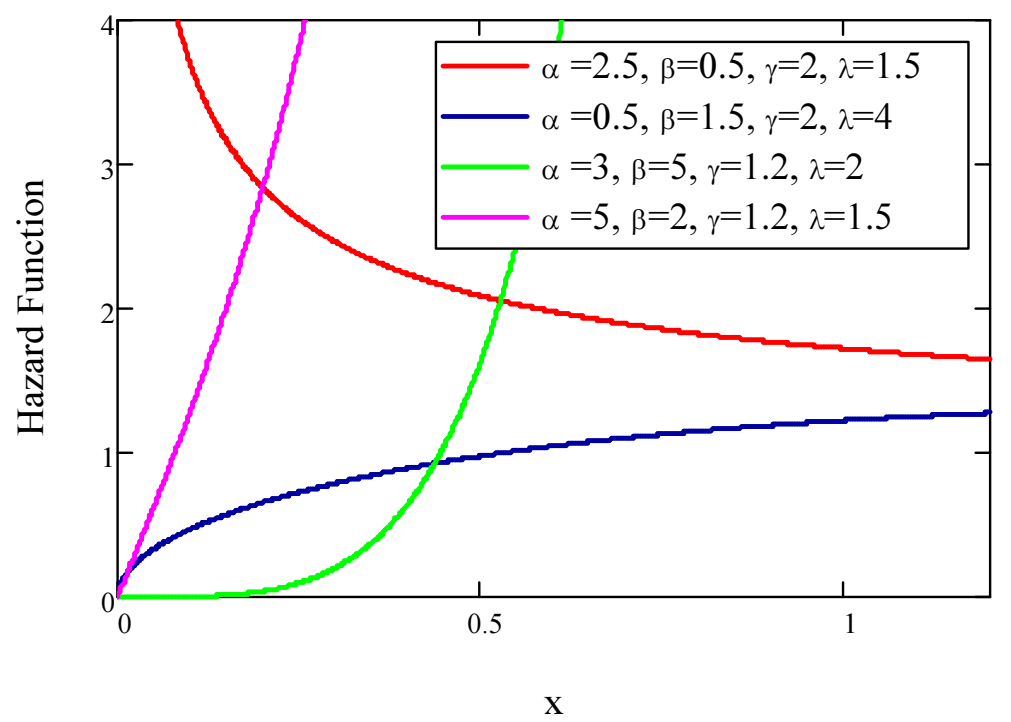

Fig. 2: Plots of hrf of OGE-PL distribution for selected values of the parameters 
The reversed-hazard rate function of the OGE-PL distribution is as follows:

$$
v(x ; \alpha, \beta, \gamma, \lambda)=\frac{\alpha \beta \lambda x^{\beta-1}\left(1+\frac{x^{\beta}}{\gamma}\right)^{\alpha-1} e^{-\lambda\left(\left(1+\frac{x^{\beta}}{\gamma}\right)^{\alpha}-1\right)}}{\gamma\left(1-e^{\left.-\lambda\left(1+\frac{x^{\beta}}{\gamma}\right)^{\alpha}-1\right)}\right)} .
$$

Additionaly, the cumulative hazard rate function of the OGE-PL is given by:

$$
H(x ; \alpha, \beta, \gamma, \lambda)=\lambda\left(\left(1+\frac{x^{\beta}}{\gamma}\right)^{\alpha}-1\right) .
$$

\section{Some Mathematical Properties}

In this section, some mathematical properties of the OGE-PL distribution including, quantiles, moments, incomplete moments, order statistics and Rényi entropy measure are derived.

\section{Quantiles of the OGE-PL Distribution}

The quantile function of the OGE-PL is given by:

$$
x_{q}=\left(\gamma\left[\left(\frac{\ln (1-q)}{-\lambda}+1\right)^{\frac{1}{\alpha}}-1\right]\right)^{\frac{1}{\beta}}, 0<q<1 \text {, }
$$

where, $q$ is a uniform $(0,1)$ distribution. We can generate random numbers from our model by using (11) and special cases can be obtained using (11) such as the second quartile (median), when $q=0.5$ :

$$
\text { Median }=x_{\text {median }}=\left(\gamma\left[\left(\frac{\ln 0.5}{-\lambda}+1\right)^{\frac{1}{\alpha}}-1\right]\right)^{\frac{1}{\beta}},
$$

and the Inter-Quantile Range (IQR) which defined as the difference between the third quartile and the first quartile can be expressed as:

$$
\begin{aligned}
\operatorname{IQR}(X) & =\left(\gamma\left[\left(\frac{\ln 0.25}{-\lambda}+1\right)^{\frac{1}{\alpha}}-1\right]\right)^{\frac{1}{\beta}} \\
& -\left(\gamma\left(\left(\frac{\ln 0.75}{-\lambda}+1\right)^{\frac{1}{\alpha}}-1\right]\right)^{\frac{1}{\beta}} .
\end{aligned}
$$

\section{Moments}

The $r$ th moment of OGE-PL is defined as: $\mu_{r}^{\prime}=\sum_{i=0}^{\infty} \sum_{j=0}^{i} \sum_{k=0}^{i+j} \eta_{i, j, k} \int_{0}^{\infty} x^{r} g(x ; \alpha(k-1), \beta, \gamma) d x, \quad r=1,2,3, \ldots$

Then, the rth moment of OGE-PL is derived as follows:

$$
\mu_{r}^{\prime}=\sum_{i=0}^{\infty} \sum_{j=0}^{i} \sum_{k=0}^{i+j} \frac{\eta_{i, j, k} \alpha(k-1) \gamma^{\frac{r}{\beta}} \Gamma\left(\alpha(k-1)-\frac{r}{\beta}\right) \Gamma\left(\frac{r+\beta}{\beta}\right)}{\Gamma(1+\alpha(k-1))} .
$$

In particular, the mean and variance of the OGE-PL distribution are given respectively by:

$$
E(X)=\sum_{i=0}^{\infty} \sum_{j=0}^{i} \sum_{k=0}^{i+j} \frac{\eta_{i, j, k} \alpha(k-1) \gamma^{\frac{1}{\beta}} \Gamma\left(\alpha(k-1)-\frac{1}{\beta}\right) \Gamma\left(\frac{1+\beta}{\beta}\right)}{\Gamma(1+\alpha(k-1))},
$$

and:

$$
\begin{aligned}
\operatorname{var}(X) & =\sum_{i=0}^{\infty} \sum_{j=0}^{i} \sum_{k=0}^{i+j} \frac{\eta_{i, j, k} \alpha(k-1) \gamma^{\frac{2}{\beta}} \Gamma\left(\alpha(k-1)-\frac{2}{\beta}\right) \Gamma\left(\frac{2+\beta}{\beta}\right)}{\Gamma(1+\alpha(k-1))} \\
& -\left[\sum_{i=0}^{\infty} \sum_{j=0}^{i} \sum_{k=0}^{i+j} \frac{\eta_{i, j, k} \alpha(k-1) \gamma^{\frac{1}{\beta}} \Gamma\left(\alpha(k-1)-\frac{1}{\beta}\right) \Gamma\left(\frac{1+\beta}{\beta}\right)}{\Gamma(1+\alpha(k-1))}\right]^{2} .
\end{aligned}
$$

The skewness $\left(g_{1}\right)$ of the OGE-PL distribution is given by:

$$
g_{1}=\frac{\mu_{3}^{\prime}-3 \mu_{1}^{\prime} \mu_{2}^{\prime}+2\left(\mu_{1}^{\prime}\right)^{3}}{\left(\mu_{2}^{\prime}-\mu_{1}^{\prime 2}\right)^{\frac{3}{2}}} .
$$

The kurtosis $\left(g_{2}\right)$ of the OGE-PL is given by:

$$
g_{2}=\frac{\mu_{4}}{\mu_{2}^{2}}=\frac{\mu_{4}^{\prime}-4 \mu_{3}^{\prime} \mu_{1}^{\prime}+6 \mu_{2}^{\prime} \mu_{1}^{\prime 2}-3 \mu_{1}^{\prime 4}}{\left(\mu_{2}^{\prime}-\mu_{1}^{\prime 2}\right)^{2}} .
$$

Furthermore, the moment generating function of OGE-PL is obtained through the following relation as follows:

$$
\begin{aligned}
M_{x}(t) & =\sum_{r=0}^{\infty} \frac{t^{r}}{r !} E\left(X^{r}\right) \\
& =\sum_{r, i=0}^{\infty} \sum_{j=0}^{i} \sum_{k=0}^{i+j} \frac{\eta_{i, j, k} \alpha(k-1) \gamma^{\frac{r}{\beta}} t^{r} \Gamma\left(\alpha(k-1)-\frac{r}{\beta}\right) \Gamma\left(\frac{r+\beta}{\beta}\right)}{r ! \Gamma(1+\alpha(k-1))}, r=1,2, \ldots
\end{aligned}
$$

\section{Incomplete Moments}

The main application of the first incomplete moment refers to the Bonferroni and Lorenz curves. These curves 
are very useful in economics, reliability, demography, insurance and medicine. The incomplete moments, say $\varphi_{s}(t)$, is given by:

$$
\varphi_{s}(t)=\int_{0}^{t} x^{s} f(x ; \Psi) d x
$$

where, $\Psi=(\alpha, \beta, \gamma, \lambda)$ is the set of parameters. Using (10), then $\varphi_{s}(t)$ can be written as follows:

$\varphi_{s}(t)=\sum_{i=0}^{\infty} \sum_{j=0}^{i} \sum_{k=0}^{i+j} \omega_{i, j, k} \gamma^{\frac{s}{\beta}} \mathrm{B}\left(\frac{s}{\beta}+1, \alpha(k-1), \frac{t^{\beta}}{\gamma+t^{\beta}}\right)$,

where, $\omega_{i, j, k}=\alpha(k-1) \eta_{i, j, k}$ and $B\left(\frac{s}{\beta}+1, \alpha(k-1), \frac{t^{\beta}}{\gamma+t^{\beta}}\right)$ is the incomplete beta function. In particular, the first incomplete moment of the OGE-PL distribution can be obtained by putting $s=1$ in (12), as follows:

$$
\varphi_{1}(t)=\sum_{i=0}^{\infty} \sum_{j=0}^{i} \sum_{k=0}^{i+j} \omega_{i, j, k} \gamma^{\frac{1}{\beta}} \mathrm{B}\left(\frac{1}{\beta}+1, \alpha(k-1), \frac{t^{\beta}}{\gamma+t^{\beta}}\right) .
$$

Additionally, the mean deviations of $X$ about the mean $\mu$ and about the median $m$ can be calculated by the following relations:

$$
\begin{aligned}
& \delta_{1}(X)=2 \mu F(\mu)-2 \varphi_{1}(\mu) \\
& \text { and } \\
& \delta_{2}(X)=\mu-2 \varphi_{1}(m),
\end{aligned}
$$

where, $\varphi_{1}($.$) is the first incomplete moment of the OGE-$ PL distribution which can be obtained from (13). By using (13) then:

$$
\varphi_{1}(\mu)=\sum_{i=0}^{\infty} \sum_{j=0}^{i} \sum_{k=0}^{i+j} \omega_{i, j, k} \gamma^{\frac{1}{\beta}} \mathrm{B}\left(\frac{1}{\beta}+1, \alpha(k-1), \frac{\mu^{\beta}}{\gamma+\mu^{\beta}}\right),
$$

and:

$$
\varphi_{1}(m)=\sum_{i=0}^{\infty} \sum_{j=0}^{i} \sum_{k=0}^{i+j} \omega_{i, j, k} \gamma^{\frac{1}{\beta}} \mathrm{B}\left(\frac{1}{\beta}+1, \alpha(k-1), \frac{m^{\beta}}{\gamma+m^{\beta}}\right) .
$$

\section{Rényi Entropy}

The entropy is a measure of the uncertainty variation. So, the Rényi entropy of a random variable $X$ with density function $f(x ; \Psi), \Psi=(\alpha, \beta, \gamma, \lambda)$ is defined as:

$$
I_{R}(X)=(1-\rho)^{-1} \ln \left\{\int_{-\infty}^{\infty} f^{\rho}(x ; \Psi) d x\right\}, \rho>0 \text { and } \rho \neq 1 .
$$

Substituting $f^{\rho}(x ; \Psi)$ in (14), then we obtain:

$$
I_{R}(X)=(1-\rho)^{-1} \ln \int_{0}^{\infty}\left(\frac{\alpha \beta \lambda}{\gamma}\right)^{\rho} x^{\rho(\beta-1)}
$$

Applying the binomial and exponential expansions in (15), then we have:

$$
I_{R}(X)=\frac{1}{1-\rho} \ln \left\{\begin{array}{l}
\sum_{i=0}^{\infty} \sum_{j=0}^{i} \sum_{k=o}^{i+j} \eta_{i, j, k} \rho^{i}(\alpha \beta \lambda)^{\rho} \gamma^{-\rho} \\
\int_{0}^{\infty} x^{\rho(\beta-1)}\left(1+\frac{x^{\beta}}{\gamma}\right)^{\rho(\alpha-1)-\alpha k} d x
\end{array}\right\},
$$

Therefore, the Rényi entropy of OGE-PL distribution is given by:

$$
I_{R}(X)=\frac{1}{1-\rho} \ln \left\{\begin{array}{l}
\sum_{i=0}^{\infty} \sum_{j=0}^{i} \sum_{k=o}^{i+j} \eta_{i, j, k} \rho^{i} \beta^{\rho-1}(\alpha \lambda)^{\rho} \gamma^{\frac{\rho(\beta-1)+1}{\beta}} \\
\mathrm{B}\left(\frac{\rho(\beta-1)+1}{\beta}, \rho(\alpha-1)-\alpha k-\frac{\rho(\beta-1)+1}{\beta}\right)
\end{array}\right\} .
$$

\section{Order Statistics}

Let $X_{1: n}<X_{2: n}<\ldots<X_{n: n}$ denote the order statistics for a random sample $X_{1}, X_{2}, \ldots, X_{n}$ from OGE-PL distribution with cdf (4) and pdf (5). The pdf of $r^{\text {th }}$ order statistics is defined by:

$$
\begin{aligned}
& f_{r: n}(x)=\frac{1}{B(r, n-r+1)} \\
& f(x ; \Psi)[F(x ; \Psi)]^{r-1}[1-F(x ; \Psi)]^{n-r}
\end{aligned}
$$

$$
\begin{gathered}
\text { By using binomial expansion in } \\
f_{r: n}(x)=\frac{1}{B(r, n-r+1)} \sum_{j=0}^{n-r}\left(\begin{array}{c}
n-r \\
j
\end{array}\right)(-1)^{j} f(x ; \Psi)[F(x ; \Psi)]^{j+r-1}
\end{gathered}
$$
have:

Inserting cdf (4) and pdf (5) in previous equation, we

$$
\begin{aligned}
& f_{r: n}(x)=\frac{1}{B(r, n-r+1)} \\
& \left.\sum_{j=0}^{n-r}\left(\begin{array}{c}
n-r \\
j
\end{array}\right)(-1)^{j} \frac{\alpha \beta \lambda}{\gamma} x^{\beta-1}\left(1+\frac{x^{\beta}}{\gamma}\right)^{\alpha-1} e^{-\lambda\left(\left(1+\frac{x^{\beta}}{\gamma}\right)^{\alpha}-1\right.}\right) \\
& {\left[1-\exp \left(-\lambda\left(\left(1+\frac{x^{\beta}}{\gamma}\right)^{\alpha}-1\right)\right)\right]^{j+r-1} .}
\end{aligned}
$$


Hence, the pdf of $r^{\text {th }}$ order statistics will be as follows:

$$
\begin{aligned}
& f_{r: n}(x)=\frac{1}{B(r, n-r+1)} \\
& \sum_{j=0}^{n-r+j+r-1}(-1)^{j+i}\left(\begin{array}{c}
n-r \\
j
\end{array}\right)\left(\begin{array}{c}
j+r-1 \\
i
\end{array}\right) \\
& \frac{\alpha \beta \lambda}{\gamma} x^{\beta-1}\left(1+\frac{x^{\beta}}{\gamma}\right)^{\alpha-1} e^{-\lambda(i+1)\left(\left(1+\frac{x^{\beta}}{\gamma}\right)^{\alpha}-1\right.} .
\end{aligned}
$$

In particular, the pdf of the smallest order statistics is obtained by substituting $r=1$ in (17) as follows:

$$
\begin{aligned}
& f_{1: n}(x)=n \sum_{j=0}^{n-1} \sum_{i=0}^{j}(-1)^{j+i}\left(\begin{array}{c}
n-1 \\
j
\end{array}\right)\left(\begin{array}{l}
j \\
i
\end{array}\right) \frac{\alpha \beta \lambda}{\gamma} \\
& x^{\beta-1}\left(1+\frac{x^{\beta}}{\gamma}\right)^{\alpha-1} e^{-\lambda(i+1)\left(\left(1+\frac{x^{\beta}}{\gamma}\right)^{\alpha}-1\right)} .
\end{aligned}
$$

Also, the pdf of largest order statistics is obtained by substituting $r=n$ in (17) as follows:

$$
f_{n: n}(x)=n \sum_{i=0}^{n-1}(-1)^{i}\left(\begin{array}{c}
n-1 \\
i
\end{array}\right) \frac{\alpha \beta \lambda}{\gamma} x^{\beta-1}\left(1+\frac{x^{\beta}}{\gamma}\right)^{\alpha-1} e^{-\lambda(i+1)\left(\left(1+\frac{x^{\beta}}{\gamma}\right)^{\alpha}-1\right)} .
$$

\section{Parameter Estimation}

In this section, the estimators of unknown parameters of the OGE-PL model are obtained based on maximum likelihood (ML) method. Let $X_{1}, X_{2}, \ldots, X_{n}$, be observed values from the OGE-PL distribution. The total loglikelihood function, denoted by $\ln L$, for the parameters $\lambda$, $\alpha, \beta$ and $\gamma$ for a complete sample is as follows:

$$
\begin{aligned}
& \ln L=\mathrm{n} \ln \alpha+\mathrm{n} \ln \beta+\mathrm{n} \ln \lambda-n \ln \gamma+(\beta-1) \\
& \sum_{i=1}^{n} \ln x_{i}+(\alpha-1) \sum_{i=1}^{n} \ln \left(1+\frac{x_{i}^{\beta}}{\gamma}\right)-\lambda \sum_{i=1}^{n}\left[\left(1+\frac{x_{i}^{\beta}}{\gamma}\right)^{\alpha}-1\right] .
\end{aligned}
$$

The partial derivatives of the log-likelihood function with respect to $\lambda, \alpha, \beta$ and $\gamma$ components of the score vector $U_{L}=\left(U_{\lambda}, U_{\alpha}, U_{\beta}, U_{\gamma}\right)^{T}$ can be obtained as follows:

$$
\begin{gathered}
U_{\lambda}=\frac{\partial \ln L}{\partial \lambda}=\frac{n}{\lambda}-\sum_{i=1}^{n}\left[\left(1+\frac{x_{i}^{\beta}}{\gamma}\right)^{\alpha}-1\right], \\
U_{\alpha}=\frac{\partial \ln L}{\partial \alpha}=\frac{n}{\alpha}+\sum_{i=1}^{n} \ln \left(1+\frac{x_{i}^{\beta}}{\gamma}\right)-\lambda \sum_{i=1}^{n}\left[\left(1+\frac{x_{i}^{\beta}}{\gamma}\right)^{\alpha} \ln \left(1+\frac{x_{i}^{\beta}}{\gamma}\right)\right], \\
U_{\beta}=\frac{n}{\beta}+\sum_{i=1}^{n} \ln x_{i}+(\alpha-1) \sum_{i=1}^{n} \frac{x_{i}^{\beta} \ln x_{i}}{\gamma+x_{i}^{\beta}}-\lambda \sum_{i=1}^{n} \alpha\left(\frac{1}{\gamma}\right)^{\alpha}\left(\gamma+x_{i}^{\beta}\right)^{\alpha-1} x_{i}^{\beta} \ln x_{i},
\end{gathered}
$$

and:

$$
U_{\gamma}=\frac{-n}{\gamma}-(\alpha-1) \sum_{i=1}^{n} \frac{x_{i}^{\beta}}{\left(\gamma^{2}+\gamma x_{i}^{\beta}\right)}+\lambda \sum_{i=1}^{n} \frac{\alpha x_{i}^{\beta}}{\gamma^{2}}\left(1+\frac{x_{i}^{\beta}}{\gamma}\right)^{\alpha-1}
$$

Then the maximum likelihood estimates (MLEs) of the parameters, denoted by $\hat{\lambda}, \hat{\alpha}, \hat{\beta}$ and $\hat{\gamma}$ are obtained by setting $U_{\lambda}, U_{\alpha}, U_{\beta}$ and $U_{\gamma}$ to be zero and solving them numerically. It is clear that, there is no closed solution for the above non-linear equations, so an extensive numerical solution will be applied via iterative technique.

\section{Data Analysis}

In this section, two real data sets are analyzed to illustrate the flexibility of OGE-PL distribution compared with some other models, namely, exponential (E), extended Poisson-Lomax (Ext.PL), Exponential Lomax (Exp Lomax), transmuted exponentiated Lomax (TE-Lomax), McDonald Lomax (McLomax), exponentiated Lomax Poisson (ELP), exponentiated Lomax (EL) and Lomax (L) distributions.

The first data set is taken from Lee and Wang (2003) referred to remission times (in months) of a random sample of 128 bladder cancer patients given as follows:

$0.08,2.09,3.48,4.87,6.94,8.66,13.11,23.63,0.20$, $2.23,3.52,4.98,6.97,9.02,13.29,0.40,2.26,3.57,5.06$, $7.09,9.22,13.80,25.74,0.50,2.46,3.64,5.09,7.26$, $9.47,14.24,25.82,0.51,2.54,3.70,5.17,7.28,9.74$, $14.76,26.31,0.81,2.62,3.82,5.32,7.32,10.06,14.77$, $32.15,2.64,3.88,5.32,7.39,10.34,14.83,34.26,0.90$, $2.69,4.18,5.34,7.59,10.66,15.96,36.66,1.05,2.69$, $4.23,5.41,7.62,10.75,16.62,43.01,1.19,2.75,4.26$, $5.41,7.63,17.12,46.12,1.26,2.83,4.33,5.49,7.66$, $11.25,17.14,79.05,1.35,2.87,5.62,7.87,11.64,17.36$, $1.40,3.02,4.34,5.71,7.93,11.79,18.10,1.46,4.40,5.85$, $8.26,11.98,19.13,1.76,3.25,4.50,6.25,8.37,12.02$, $2.02,3.31,4.51,6.54,8.53,12.03,20.28,2.02,3.36,6.76$, $12.07,21.73,2.07,3.36,6.93,8.65,12.63,22.69$.

The MLEs for OGE-PL, Ext.PL, Exp Lomax, McLomax, TE-Lomax, L and EL models are given in Table 1. Also, to compare the distribution models, we consider criteria like; Akaike information criterion (AIC), the Correct Akaike Information Criterion (CAIC), Bayesian Information Criterion (BIC) and Hannan-Quinn information criterion (HQIC). However, the better distribution corresponds to the smaller values of AIC, BIC, CAIC, HQIC criteria. These criteria are provided in Table 2.

It is clear from Table 2 that the OGE-PL distribution provides a better fit than the other competitive models. It has the smallest AIC, CAIC, BIC and HQIC values among those considered here. 
Table 1: MLEs of the model parameters for the 128 remission times (in months) of bladder cancer patient's data

\begin{tabular}{lllllllll}
\hline Distribution & $\hat{\alpha}$ & $\hat{\beta}$ & $\hat{\gamma}$ & $\hat{\lambda}$ & $\hat{\theta}$ & $\hat{a}$ & $\hat{b}$ & $\hat{c}$ \\
\hline OGE-PL & 0.136 & 1.453 & 25.382 & 10.941 & & - & - & - \\
Ext.PL & 0.239 & $8.04 \times 10^{3}$ & 59.838 & - & - & - & - \\
Exp Lomax & 1.0644 & 0.08 & 0.006 & - & & - & - & - \\
McLomax & 0.8085 & 11.2929 & - & - & & 1.506 & 4.1886 & 2.1046 \\
TE-Lomax & 1.714 & & 0.054 & 0.244 & 3.339 & - & - & - \\
EL & 131.499 & 197.476 & 0.756 & - & & - & - & - \\
L & 13.938 & - & 121.023 & - & & - & - & - \\
\hline
\end{tabular}

Table 2: The statistics AIC, CAIC, BIC and HQIC for the 128 remission times (in months) of bladder cancer patients data

\begin{tabular}{lllll}
\hline Distribution & AIC BIC & CAIC & $H Q I C$ & 832.093 \\
\hline OGE-PL & 827.458 & 838.866 & 827.783 & 837.140 \\
Ext.PL & 833.670 & 842.220 & 833.860 & 839.432 \\
Exp Lomax & 835.956 & 844.512 & 836.150 & 835.620 \\
McLomax & 829.820 & 844.090 & 830.140 & 833.505 \\
TE-Lomax & 828.868 & 840.276 & 829.130 & 897.892 \\
EL & 894.416 & 902.972 & 894.609 & 833.980 \\
L & 831.670 & 837.370 & 831.800 & \\
\hline
\end{tabular}

Table 3: The MLEs of the model parameters and the statistics AIC, BIC, CAIC and HQIC for failure times of 84 Aircraft Windshield data

\begin{tabular}{|c|c|c|c|c|c|}
\hline Distribution & Estimates & $A I C$ & $B I C$ & CAIC & $H Q I C$ \\
\hline OGE-PL & $\begin{array}{l}a=4.752 \\
\beta=0.888 \\
\gamma=1.618 \\
\lambda=0.01\end{array}$ & 261.448 & 271.172 & 261.955 & 265.357 \\
\hline ELP & $\begin{array}{l}a=2.666 \\
\beta=0.005 \\
\gamma=17.628 \\
\lambda=49.961\end{array}$ & 273.832 & 283.602 & 274.332 & 277.741 \\
\hline McLomax & $\begin{array}{l}a=2.792 \\
b=13.802 \\
c=3.907 \\
\alpha=2.230 \\
\beta=0.113\end{array}$ & 280.048 & 292.261 & 280.807 & 282.002 \\
\hline EL & $\begin{array}{l}\alpha=4.325 \\
\beta=3.184 \\
\gamma=0.329\end{array}$ & 313.950 & 321.278 & 314.246 & 316.882 \\
\hline $\mathrm{L}$ & $\begin{array}{l}\alpha=28.329 \\
\gamma=0.014\end{array}$ & 336.398 & 341.283 & 336.544 & 338.352 \\
\hline E & $\lambda=0.389$ & 328.501 & 330.932 & 328.549 & 329.478 \\
\hline
\end{tabular}

As a second example, the data set recently studied by Ramos et al. (2013) will be considered. It consists of the failure times of 84 Aircraft Windshield (the unit for measurement is 1000 hours) is given as follows:

$0.040,1.866,2.385,3.443,0.301,1.876,2.481,3.467$, $0.309,1.899,2.610,3.478,0.557,1.911,2.625,3.578$, $0.943,1.912,2.632,3.595,1.070,1.914,2.646,3.699$, $1.124,1.981,2.661,3.779,1.248,2.010,2.688,3.924$, $1.281,2.038,2.82,3,4.035,1.281,2.085,2.890,4.121$, $1.303,2.089,2.902,4.167,1.432,2.097,2.934,4.240$, $1.480,2.135,2.962,4.255,1.505,2.154,2.964,4.278$, $1.506,2.190,3.000,4.305,1.568,2.194,3.103,4.376$,
$1.615,2.223,3.114,4.449,1.619,2.224,3.117,4.485$, $1.652,2.229,3.166,4.570,1.652,2.300,3.344,4.602$, $1.757,2.324,3.376,4.663$.

The required numerical evaluations are carried out using the package of Mathcad software. The MLEs for OGE-PL, ELP, EL, McLomax, L and E models and the values of the $A I C, B I C, C A I C$ and $H Q I C$ are listed in Table 3.

It is clear from Table 3 that the proposed OGE-PL distribution fits to this data is better than the other models according to the AIC, BIC, CAIC and HQIC. 


\section{Conclusion}

In this article, we propose a new model, called the odds generalized exponential-Power Lomax distribution based on T-X family presented by Alzaatreh et al. (2013). Some statistical properties of current distribution have been derived and discussed. The estimation of the model parameters is approached by maximum likelihood method. We fit the OGE-PL distribution to two real life data sets as two examples, the OGE-PL distribution provides consistently a better fit than some other known models.

\section{Acknowledgment}

The author would like to thank Prof. Amal Soliman Hassan for her careful comments which significantly improved the paper. Also, the author would like to thank the Editor and an anonymous referee for their valuable suggestions which led to an improved version of the original paper.

\section{Ethics}

The author declare that it is my original work and has not been considered for publication in whole or in part elsewhere.

\section{References}

Abdul-Moniem, I.B., 2012. Recurrence relations for moments of lower generalized order statistics from exponentiated Lomax distribution and its characterization. J. Mathematical Computational Sci., 2: 999-1011.

Alzaatreh, A., C. Lee and F. Famoye, 2013. A new method for generating families of continuous distributions. Metron, 71: 63-79.

DOI: $10.1007 / \mathrm{s} 40300-013-0007-y$

Al-Zahrani, B., 2015. An extended Poisson-Lomax distribution. Advances Mathematics: Scientific J., 4: 79-89.

Ashour, S. and M. Eltehiwy, 2013. Transmuted exponentiated Lomax distribution. Australian J. Basic Applied Sci., 7: 658-667.

Cordeiro, G.M., E.M.M. Ortega and B.V. Popovi'c, 2013. The gamma-Lomax distribution. J. Statistical Computation Simulation.

DOI: $10.1080 / 00949655.2013 .822869$

El-Bassiouny, A.H., N.F. Abdo and H.S. Shahen, 2015. Exponential Lomax distribution. Int. J. Computer Applications, 121: 24-29.

Ghitany, M.E., F.A. AL-Awadhi and L.A. Alkhalfan, 2007. Marshall-Olkin extended Lomax distribution and its applications to censored data. Communications Statistics-Theory Methods, 36: 1855-1866. DOI: 10.1080/03610920601126571
Gupta, R.C., M.E. Ghitany and D.K. Al-Mutairi, 2010. Estimation of reliability from Marshall-Olkin extended Lomax distributions. J. Statistical Computation Simulation, 80: 937-947. DOI: $10.1080 / 00949650902845672$

Haq, M.A. and M. Elgarhy, 2018. The odd Frèchet-G family of probability distributions. J. Statistics Applications Probability, 7: 189-203.

Hassan, A.S. and A.S. Al-Ghamdi, 2009. Optimum step stress accelerated life testing for Lomax distribution. J. Applied Sciences Res., 5: 2153-2164.

Hassan, A.S. and M. Abd-Alla, 2018. Exponentiated Weibull Lomax: Properties and estimation. J. Data Sci., 16: 277-298.

Hassan, A.S., E.A. Elsherpieny and R.E. Mohamed, 2018. Odds generalized exponential-inverse Weibull distribution: properties and estimation. Pakistan J. Statistics Operation Res., 14: 1-22. DOI: $10.18187 /$ pjsor.v14i1.2086

Hosseini, B., M. Afshari and M. Alizadeh, 2018. The generalized odd gamma-G family of distributions: Properties and applications. Austrian J. Statistics, 47: 69-89. DOI: 10.17713/ajs.v47i2.580

Lee, E.T. and J.W. Wang, 2003. Statistical Methods for Survival Data Analysis. 3rd Edn., Wiley, New York.

Lemonte, A.J. and G.M. Cordeiro, 2013. An extended Lomax distribution. Statistics, 47: 800-816. DOI: $10.1080 / 02331888.2011 .568119$

Lomax, K.S., 1954. Business failures: Another example of the analysis of failure data. J. Am. Statistical Association, 49: 847-852. DOI: $10.1080 / 01621459.1954 .10501239$

Rady, E.A., W.A. Hassanein and T.A. Elhaddad, 2016. The power Lomax distribution with an application to bladder data. Springerplus, 5: 1838 . DOI 10.1186/s40064-016-3464-y

Ramos, M.W.A., P.R.D. Marinho, R.V. da Silva and G.M. Cordeiro, 2013. The exponentiated Lomax Poisson distribution with an application to lifetime data. Advances Applications Statistics, 34: 107-135.

Rosaiah, K., G.S. Rao, D.C.U. Sivakumar and K. Kalyani, 2016. The odd generalized exponential log logistic distribution. Int. J. Math. Statistics Invention, 4: 21-29.

Silva, F.G., A. Percontini, E. Brito, M.W. Ramos and R. Venâncio et al., 2017. The odd Lindley-G family of distributions. Austrian J. Statistics, 46: 65-87. DOI: 10.17713/ajs.v46i1.222

Tahir, M.H., G.M. Cordeiro, M. Mansoor and M. Zubair, 2015a. The Weibull-Lomax distribution: properties and applications. Hacettepe J. Math. Statistics, 44: 461-480.

Tahir. M.H., G.M. Cordeiro, M. Alizadeh, M. Mansoor and M. Zubair et al., 2015b. The odd generalized exponential family of distributions with applications. J. Statistical Distributions Applications, 2: 1-28. DOI: $10.1186 / \mathrm{s} 40488-014-0024-2$ 